\title{
Clinical Outcomes in Patients Treated With a Repositionable and Fully Retrievable Aortic Valve - REPRISE Japan Study -
}

\author{
Shigeru Saito, MD; Kentaro Hayashida, MD, PhD; Morimasa Takayama, MD, PhD; \\ Tsuyoshi Goto, MD; Leo Ihlberg, MD, PhD; Yoshiki Sawa, MD, PhD
}

\begin{abstract}
Background: The REPRISE Japan study, a prospective multicenter single-arm trial, was undertaken to confirm the safety and effectiveness of transcatheter aortic valve replacement (TAVR) with the LOTUS valve in Japanese subjects with severe symptomatic calcific aortic stenosis at extreme or high surgical risk.
\end{abstract}

Methods and Results: REPRISE Japan enrolled 40 subjects in the transfemoral (TF) cohort (mean age 84 years; mean [ \pm SD] Society of Thoracic Surgeons [STS] score 6.4 $\pm 2.9 \%$ ); 10 additional subjects were treated with a transaortic (TAo) approach (mean age 84 years; mean STS score $6.3 \pm 3.3 \%$ ). A subanalysis was also performed on subjects treated with the 21-mm LOTUS valve $(n=15$; mean age 84 years; mean STS score $5.3 \pm 2.1 \%)$. The primary safety endpoint (a composite of all-cause mortality, stroke, life-threatening or major bleeding events, acute kidney injury [Stage 2/3], and major vascular complications at 30 days) occurred in $15 \%$ of TF subjects. The primary effectiveness endpoint (a composite of all-cause mortality, disabling stroke, and moderate or greater paravalvular leak [PVL; core laboratory assessed] at 6 months) occurred in 5.3\% of TF subjects. Across the TF, TAo, and 21 -mm LOTUS valve cohorts, no subjects exhibited moderate or greater PVL at 6 months. The 30-day rate of pacemaker implantation was $22.5 \%$ in the TF cohort (TAo: $20 \%$; $21 \mathrm{~mm}: 13.3 \%$ ).

Conclusions: Data from REPRISE Japan confirm the safety and efficacy of the LOTUS Valve when used in Japanese clinical practice.

Key Words: Aortic regurgitation; Aortic valve stenosis; Transcatheter aortic valve replacement; Transfemoral

$\mathbf{T}$ ranscatheter aortic valve replacement (TAVR) is a safe and effective alternative to surgical valve replacement for high-risk subjects with symptomatic severe aortic stenosis. ${ }^{1-4}$ Most TAVR studies have been conducted in Western patients; however, TAVR is increasing rapidly in Japan.

Clinical studies and patient registries evaluating TAVR in Asian patients treated with balloon-expandable and selfexpanding valves have shown that clinical outcomes are comparable to those observed in Western patients. ${ }^{58}$ Nonetheless, some concerns remain regarding the increased risk of procedural complications related to anatomical differences between Western and Asian patients. ${ }^{7,9}$ Due to a smaller average body size, Asian patients may have reduced femoral vascular access. A smaller annular diam- eter can create problems related to accurate prosthetic valve sizing and the potential for aortic root rupture during predilatation or valve expansion. Smaller aortic sinus depth can increase the risk of valve malpositioning or embolization, coronary artery occlusion, and paravalvular leak (PVL).

The LOTUS valve (Boston Scientific, Marlborough, MA, USA) is a mechanically expanded TAVR valve with several design features that may help address concerns related to access, positioning, and sizing. The LOTUS valve offers controlled delivery via gradual mechanical expansion by either a transfemoral (TF) or transaortic (TAo) route. The LOTUS valve is repositionable and fully retrievable to ensure optimal positioning and deployment, and has an integrated sealing skirt designed to reduce PVL.

Received May 10, 2020; revised manuscript received September 3, 2020; accepted September 24, 2020; J-STAGE Advance Publication released online November 14, 2020 Time for primary review: 29 days

Department of Cardiology, Shonan Kamakura General Hospital, Kamakura (S.S.); Department of Cardiology, Keio University School of Medicine, Tokyo (K.H.); Department of Cardiology, Sakakibara Heart Institute, Tokyo (M.T.); Department of Cardiology, Kurashiki Central Hospital, Kurashiki (T.G.), Japan; Boston Scientific Corporation, Marlborough, MA (L.I.), USA; and Department of Cardiovascular Surgery, Osaka University Graduate School of Medicine, Osaka (Y.S.), Japan

REPRISE Japan Study is registered with ClinicalTrials.gov (ID: NCT02491255).

Mailing address: Shigeru Saito, MD, Director of Cardiology and Catheterization Laboratories, Vice President Shonan Kamakura General Hospital, 1370-1 Okamoto, Kamakura 247-8533, Japan. E-mail: transradial@kamakuraheart.org

All rights are reserved to the Japanese Circulation Society. For permissions, please e-mail: cj@j-circ.or.jp

ISSN-1346-9843 


\section{The Lotus Valve System}

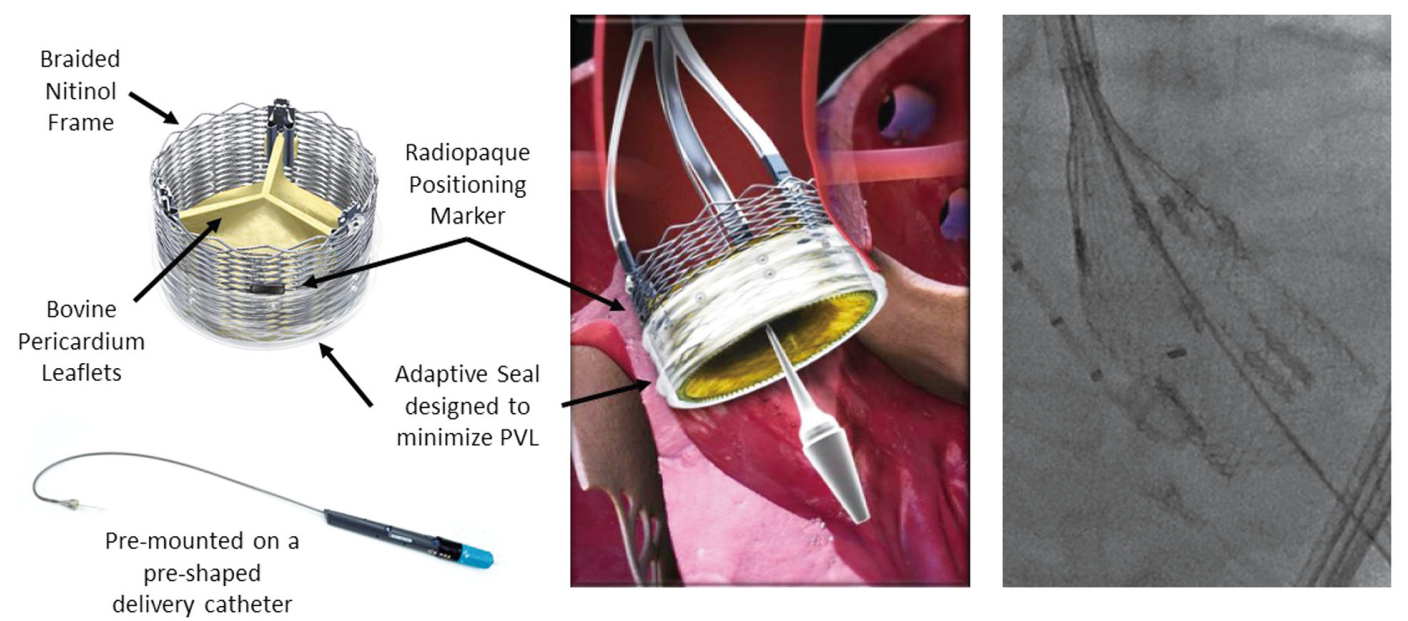

Figure 1. The LOTUS valve system. The LOTUS valve is a fully retrievable and repositionable bioprosthetic aortic valve comprised of bovine pericardial leaflets sutured to a braided nitinol wire frame mounted on a preshaped delivery catheter. The valve is deployed via controlled mechanical expansion for precise placement and functions early to enable hemodynamic stability. A polymer membrane "adaptive seal" conforms to the annulus to minimize paravalvular leak (PVL).

The REpositionable Percutaneous Replacement of Stenotic Aortic Valve through Implantation of LOTUS Valve System (REPRISE) I and II studies demonstrated the safety and efficacy of TAVR with the LOTUS valve in patients at high or extreme risk of surgical valve replacement. ${ }^{10,11}$ The recent REPRISE III randomized controlled trial demonstrated comparable safety and efficacy with the LOTUS valve and a self-expanding TAVR valve, with significantly less PVL with the LOTUS valve, albeit with a greater need for permanent pacemaker implantation (PPI).12

There have been no clinical studies to date of TAVR with the LOTUS valve in Japanese patients. Here we report primary safety and effectiveness outcomes from REPRISE Japan, the first study of the LOTUS valve in a Japanese population.

\section{Methods}

\section{Study Design and Subject Enrolment}

The REPRISE Japan study, a prospective multicenter single-arm trial, enrolled subjects with calcific severe native aortic stenosis who were at extreme or high risk for standard surgical valve replacement. The study enrolled subjects at 5 centers across Japan.

As per the study protocol, a subject must have documented calcific severe native aortic stenosis (initial aortic valve area $[\mathrm{AVA}] \leq 1.0 \mathrm{~cm}^{2}$ or an AVA index of $\leq 0.6 \mathrm{~cm}^{2} / \mathrm{m}^{2}$ ) and a mean pressure gradient $\geq 40 \mathrm{mmHg}$ or a jet velocity $\geq 4 \mathrm{~m} / \mathrm{s}$, a documented aortic annulus size of $\geq 18$ and $\leq 27 \mathrm{~mm}$ based on preprocedural diagnostic imaging, and New York Heart Association (NYHA) functional class $\geq$ II. Subjects were evaluated by a local heart team, which included the site investigator interventionalist and site investigator cardiac surgeon, to confirm high or extreme operative risk for surgical valve replacement (e.g., Society of Thoracic Surgeons [STS] score $\geq 8 \%$ or, if STS $<8 \%$, confirmation of the presence of $\geq 1$ surgical risk factors, which are detailed in full in Supplementary Table 1). Subjects with bicuspid aortic valves were excluded from the study. Eligibility was confirmed by the case review committee. Subjects must have provided written informed consent before undergoing any study-specific tests or procedures.

The main study cohort comprised subjects who were treated with a 23-, 25-, or 27-mm LOTUS valve via a TF or transiliac approach; target enrollment was 50 subjects, which included the enrollment of 2 roll-in subjects per site (only non-roll-in subjects were included in endpoint analyses). The TAo substudy enrolled subjects in whom neither a TF nor transiliac approach could be safely performed. In addition, a 21-mm substudy evaluated the use of the 21-mm LOTUS valve via the TF, transiliac, or TAo approaches.

The study protocol was approved by the central ethics committee of Mirai Iryo Research Center Inc. (Reference no. 024-14-25). The study was conducted in accordance with the International Conference on Harmonization Guidelines for Good Clinical Practice and the ethical principles outlined in the Declaration of Helsinki. The study was sponsored by Boston Scientific Corporation and registered with ClinicalTrials.gov (ID: NCT02491255). All subjects provided written informed consent.

\section{Study Device}

The LOTUS valve (Figure 1) is a bioprosthetic aortic valve comprised of a braided nitinol wire frame with 3 bovine pericardial leaflets premounted on a preshaped delivery catheter and deployed via controlled mechanical expansion. ${ }^{11,13}$ The LOTUS delivery system features an atraumatic tip design and a hydrophilic coating to facilitate sheath entry and removal and to enhance tracking through challenging anatomy; in addition, the design of the flexible, reinforced catheter provides improved resistance to kinking. 


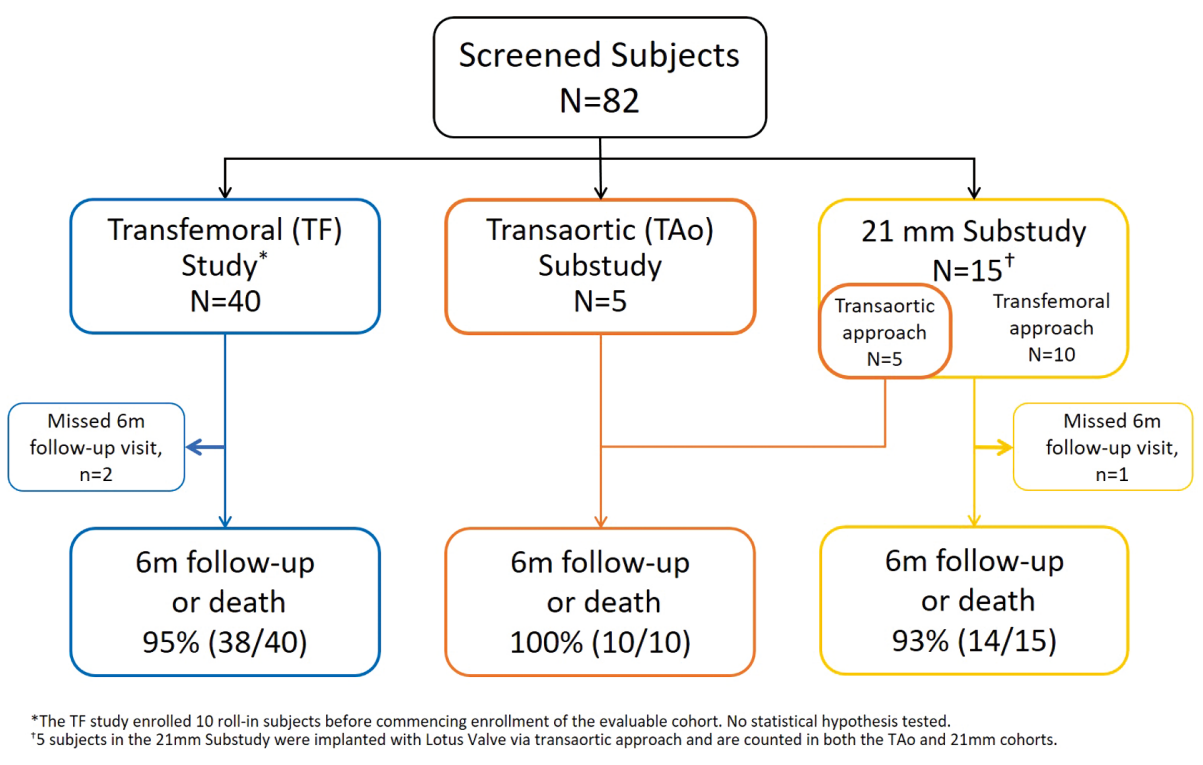

Figure 2. Study subjects. This study evaluated 40 subjects who were treated with a 23-, 25-, or 27-mm LOTUS valve via a transfemoral (TF) or transiliac approach. Subjects in whom neither a TF nor transiliac approach could be safely performed were enrolled in a transaortic (TAo) substudy $(n=5)$. In addition, 15 subjects were treated with a 21-mm LOTUS valve (TF or transiliac approach, $n=10$; TAo approach, $n=5)$.

The valve features a polymer membrane "adaptive seal" surrounding its lower half, designed to fill the space between the native annulus and the prosthetic valve frame and reduce PVL. The LOTUS valve functions early in the deployment process, providing hemodynamic stability so that rapid pacing is not required. Repositioning or retrieval of the valve is possible at any point prior to uncoupling and release. REPRISE Japan evaluated LOTUS valve sizes of $21,23,25$, and $27 \mathrm{~mm}$.

It should be noted that during the time of enrolment and follow-up in REPRISE Japan, Boston Scientific became aware of complaints related to device malfunction of the delivery system, and commercial sales of the device were stopped in regions where it had been approved. The next iteration of the device, LOTUS Edge, was the first version of the product approved for use in Japan (approval December 2019; first commercial use in Japan early 2020). LOTUS Edge features several improvements, which are described in full in the Discussion and include design modifications to ensure reliability of the delivery system.

\section{Outcomes Measures}

Endpoints were assessed according to Valve Academic Research Consortium (VARC)-2 metrics, ${ }^{14}$ with the exception of bleeding events, which were reported according to a modified Bleeding Academic Research Consortium (BARC) definition (see Supplementary Table 2). The primary safety endpoint of the study was a composite of allcause mortality, stroke, life-threatening and major bleeding events, Stage 2 or 3 acute kidney injury, or major vascular complications at 30 days. The primary effectiveness endpoint was a composite of all-cause mortality, disabling stroke, or moderate or greater PVL at 6 months. Safety events were adjudicated by an independent clinical events committee. All subjects had a neurological examination conducted by an independent neurology professional (i.e., not involved with the care of the study subjects) at baseline, discharge (or 7 days), and after any suspected stroke. In addition, the National Institutes of Health Stroke Scale (NIHSS) score was determined for all subjects at baseline and discharge or 7 days, and the modified Rankin Score (mRS) was determined at baseline, discharge or 7 days, and all follow-up time points. The degree of moderate or greater PVL at 6 months was analyzed separately as a secondary endpoint. Echocardiographic measures were based on independent core laboratory assessment (MedStar Health Research Institute, Washington, DC, USA) to minimize bias and inconsistencies.

\section{Statistical Analysis}

The analysis population for the primary safety and primary effectiveness endpoints was based on the implanted analysis set, comprising intent-to-treat subjects who were implanted with the LOTUS valve. In the TF study, endpoints were evaluated only for those subjects enrolled after the roll-in phase. Baseline and outcome variables were summarized using descriptive statistics. All $\mathrm{P}$ values are 2-sided and were derived from either a 1-sample exact binominal test (for safety endpoints), a generalized McNemar's test (for improvement in NYHA functional classification), or a paired t-test analysis (for change in hemodynamic parameters among subjects with echocardiographic data available at both specified time points). Data from the TAo and 21-mm substudies were not pooled with the main TF cohort for any analyses.

\section{Results}

\section{Study Participants and Baseline Characteristics}

The REPRISE Japan TF study enrolled 10 roll-in subjects 


\begin{tabular}{|c|c|c|c|}
\hline & $\begin{array}{l}\text { TF study } \\
(n=40)\end{array}$ & $\begin{array}{l}\text { TAo substudy } \\
(n=10)\end{array}$ & $\begin{array}{c}21-m m \text { substudy } \\
(n=15)\end{array}$ \\
\hline \multicolumn{4}{|l|}{ Demographics and comorbidities } \\
\hline Age (years) & $84 \pm 6$ & $84 \pm 6$ & $84 \pm 4$ \\
\hline Female sex & $60(24)$ & $70(7)$ & $100(15)$ \\
\hline STS score & $6.4 \pm 2.9$ & $6.3 \pm 3.3$ & $5.3 \pm 2.1$ \\
\hline STS $\geq 8 \%$ & $25(10)$ & $30(3)$ & $13(2)$ \\
\hline EuroSCORE & $4.6 \pm 4.3$ & $4.9 \pm 1.8$ & $3.9 \pm 1.3$ \\
\hline High surgical risk & $88(35)$ & $70(7)$ & $93(14)$ \\
\hline Extreme surgical risk & $13(5)$ & $30(3)$ & $7(1)$ \\
\hline $\mathrm{BMI}\left(\mathrm{kg} / \mathrm{m}^{2}\right)$ & $22.6 \pm 4.3$ & $21.9 \pm 3.8$ & $22.1 \pm 3.4$ \\
\hline Diabetes, medically treated & $22.5(9)$ & $30(3)$ & $26.7(4)$ \\
\hline CAD & $33(13)$ & $60(6)$ & $40(6)$ \\
\hline Prior PCl & $30(12)$ & $60(6)$ & $40(6)$ \\
\hline Prior CABG & $5(2)$ & $0(0)$ & $0(0)$ \\
\hline Prior MI & $5(2)$ & $20(2)$ & $20(3)$ \\
\hline Atrial fibrillation & $13(5)$ & $30(3)$ & $27(4)$ \\
\hline Pacemaker & $5(2)$ & $10(1)$ & $13(2)$ \\
\hline Prior stroke & $5(2)$ & $10(1)$ & $7(1)$ \\
\hline PVD & $8(3)$ & $50(5)$ & $13(2)$ \\
\hline COPD & $18(7)$ & $20(2)$ & $13(2)$ \\
\hline \multicolumn{4}{|l|}{ Baseline echocardiography } \\
\hline Aortic valve area $\left(\mathrm{cm}^{2}\right)$ & $0.58 \pm 0.20$ & $0.59 \pm 0.13$ & $0.55 \pm 0.16$ \\
\hline Mean aortic gradient $(\mathrm{mmHg})$ & $57.3 \pm 20.2$ & $54.3 \pm 9.5$ & $59.4 \pm 17.2$ \\
\hline Peak aortic gradient $(\mathrm{mmHg})$ & $87.0 \pm 29.9$ & $83.9 \pm 14.7$ & $91.3 \pm 27.3$ \\
\hline Aortic regurgitation moderate or greater & $10(4)$ & $10(1)$ & $20(3)$ \\
\hline Mitral regurgitation moderate or greater & 0 & 0 & 0 \\
\hline LVEF (\%) & $54.2 \pm 9.2$ & $57.0 \pm 10.2$ & $61.1 \pm 5.4$ \\
\hline \multicolumn{4}{|l|}{ Implanted valve size (mm) } \\
\hline 21 & 0 & $50(5)$ & $100(15)$ \\
\hline 23 & $52.5(21)$ & $30(3)$ & 0 \\
\hline 25 & $37.5(15)$ & $20(2)$ & 0 \\
\hline 27 & $10.0(4)$ & 0 & 0 \\
\hline \multicolumn{4}{|l|}{ Device performance } \\
\hline $\begin{array}{l}\text { Successful vascular access, delivery and } \\
\text { deployment of the LOTUS valve system, and } \\
\text { successful retrieval of delivery system }\end{array}$ & $100(40 / 40)$ & $100(10 / 10)$ & $100(15 / 15)$ \\
\hline Attempted repositioning & $42.5(17 / 40)$ & $50.0(5 / 10)$ & $33.3(5 / 15)$ \\
\hline Successful repositioning ${ }^{A}$ & $88.2(15 / 17)$ & $100(5 / 5)$ & $100(5 / 5)$ \\
\hline Successful repositioning for last valve attempted ${ }^{\mathrm{B}}$ & $100.0(16 / 16)$ & $100(5 / 5)$ & $100(5 / 5)$ \\
\hline Attempted retrieval & $7.5(3 / 40)$ & $0 / 0(0 / 10)$ & $6.7(1 / 15)$ \\
\hline Successful retrievalc & $100(3 / 3)$ & NA & $100(1 / 1)$ \\
\hline
\end{tabular}

Subjects were either treated with a 23-, 25-, or 27-mm LOTUS valve via a transfemoral or transiliac approach (TF group), enrolled in the transaortic (TAo) substudy, or treated with a 21-mm LOTUS valve. Unless indicated otherwise, data are presented as the mean \pm SD or percentages, with the number of subjects given in parentheses. Device performance data are subject based. APartial or complete resheathing of the LOTUS valve in the catheter and redeployment in a more accurate position within the aortic valve annulus. BIn 2 subjects the initial repositioning attempt was unsuccessful; in both cases, the valve was successfully retrieved and replaced with a new valve of the same size. 'Complete resheathing of the LOTUS valve in the catheter and removal from the body. BMI, body mass index; CABG, coronary artery bypass graft; CAD, coronary artery disease; COPD, chronic obstructive pulmonary disease; LVEF, left ventricular ejection fraction; $\mathrm{MI}$, myocardial infarction; $\mathrm{NA}$, not applicable; $\mathrm{PCl}$, percutaneous coronary intervention; PVD, peripheral vascular disease; STS, Society of Thoracic Surgeons.

and 40 evaluable subjects at 5 sites from June 22, 2015 to May 30, 2016. The 21-mm substudy enrolled 15 subjects at 5 sites from February 9, 2016 to August 23, 2016, all of whom were successfully treated with the LOTUS valve (TF or transiliac approach, $\mathrm{n}=10$; TAo approach, $\mathrm{n}=5$ ); the TAo substudy enrolled 5 additional TAo subjects at 3 sites from January 19, 2016 to July 12, 2016. Figure 2 illustrates subject enrollment in each cohort.

Baseline demographics and echocardiographic characteristics for all REPRISE Japan subjects are presented in Table 1. Most subjects were elderly (mean age 84 years). In the TF study, surgical risk was judged to be high in $87.5 \%$ of subjects and extreme in $12.5 \%$ of subjects. The mean $( \pm \mathrm{SD})$ STS score in the TF study was $6.4 \pm 2.9 \%$. The risk 
Table 2. Clinical Safety Outcomes at 30 Days and 6 Months

\begin{tabular}{|c|c|c|c|c|c|c|}
\hline & \multicolumn{2}{|c|}{ TF study $(n=40)$} & \multicolumn{2}{|c|}{ TAo substudy $(n=10)$} & \multicolumn{2}{|c|}{ 21-mm sub-study $(n=15)$} \\
\hline & 30 days & 6 months & 30 days & 6 months & 30 days & 6 months \\
\hline All-cause mortality* & 0 & $2.5(1)$ & 0 & $10.0(1)$ & 0 & 0 \\
\hline Cardiovascular mortality & 0 & $2.5(1)$ & 0 & $10.0(1)$ & 0 & 0 \\
\hline Stroke* & $7.5(3)$ & $7.5(3)$ & 0 & 0 & $6.7(1)$ & $6.7(1)$ \\
\hline Disabling stroke & $2.5(1)$ & $2.5(1)$ & 0 & 0 & $6.7(1)$ & $6.7(1)$ \\
\hline Life-threatening bleeding $\mathrm{A}^{*}$ & $5.0(2)$ & $5.0(2)$ & $50.0(5)$ & $50.0(5)$ & $13.3(2)$ & $13.3(2)$ \\
\hline Major bleeding* & 0 & 0 & $10.0(1)$ & $10.0(1)$ & $6.7(1)$ & $6.7(1)$ \\
\hline $\mathrm{AKI}^{*}$ (Stage $2 / 3 \leq 7$ days) & 0 & 0 & $10.0(1)$ & $10.0(1)$ & 0 & 0 \\
\hline Major vascular complications* & $2.5(1)$ & $2.5(1)$ & $20.0(2)$ & $20.0(2)$ & $6.7(1)$ & $6.7(1)$ \\
\hline Myocardial infarction & 0 & 0 & 0 & 0 & 0 & 0 \\
\hline New-onset atrial fibrillation & $5.0(2)$ & $5.0(2)$ & 0 & 0 & $6.7(1)$ & $6.7(1)$ \\
\hline $\begin{array}{l}\text { Hospitalization for valve-related symptoms or } \\
\text { worsening } \mathrm{CHF}\end{array}$ & 0 & 0 & 0 & $10.0(1)$ & 0 & 0 \\
\hline Reintervention ${ }^{B}$ & 0 & 0 & 0 & 0 & 0 & 0 \\
\hline Pacemaker implantation & $22.5(9)$ & $22.5(9)$ & $20.0(2)$ & $30.0(3)$ & $13.3(2)$ & $13.3(2)$ \\
\hline$V^{2}$ alve malpositioning ${ }^{C}$ & 0 & 0 & 0 & 0 & 0 & 0 \\
\hline Endocarditis & 0 & 0 & 0 & $10.0(1)$ & 0 & 0 \\
\hline Valve thrombosis & 0 & 0 & 0 & $10.0(1)$ & 0 & 0 \\
\hline
\end{tabular}

Subjects were either treated with a 23-, 25-, or 27-mm LOTUS valve via a transfemoral or transiliac approach (TF group), enrolled in the transaortic (TAo) substudy, or treated with a 21-mm LOTUS valve. Unless indicated otherwise, data are presented as the mean \pm SD or percentages, with the number of subjects given in parentheses. *Component of the composite primary safety endpoint. ATF cohort: upper gastrointestinal bleeding $(n=1)$, access site bleeding $(n=1)$; TAo cohort: osteorrhagia $(n=1)$, bleeding from cannulation site, hemostasis achieved through thoracotomy $(n=1)$, melena related to upper gastrointestinal bleeding $(n=1)$; TAo+21-mm cohort: aortic access site bleeding, resolved via ligation ( $n=1)$, left ventricular rupture requiring surgical intervention $(n=1)$. BRepeat procedure for valve-related dysfunction, including surgical or interventional therapy. CIncludes valve migration, valve embolization, ectopic valve deployment. AKI, acute kidney injury; CHF, congestive heart failure.

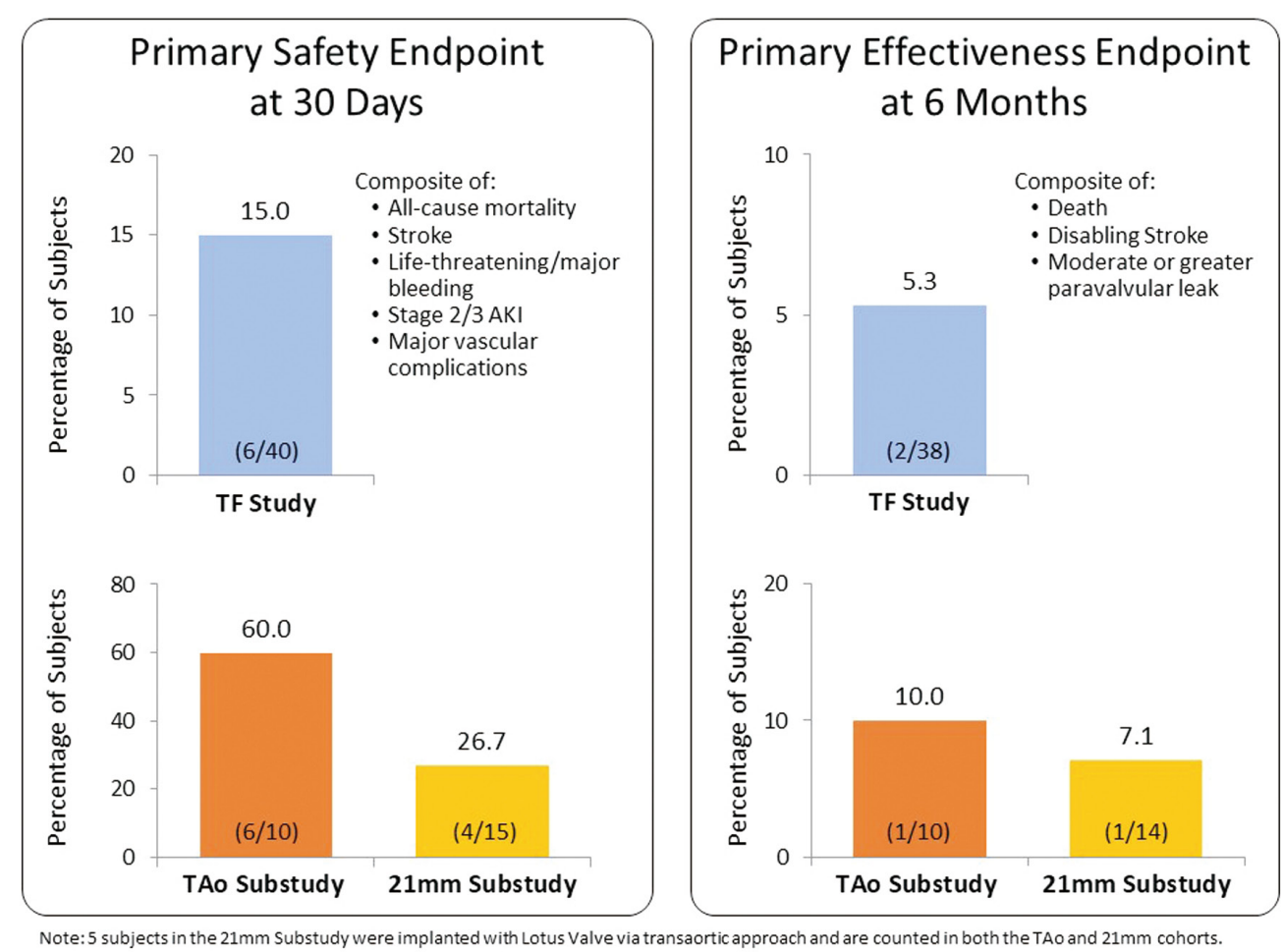

Figure 3. Primary safety and effectiveness endpoints. The primary safety endpoint was a composite of all-cause mortality, stroke, life-threatening or major bleeding events, acute kidney injury (AKI; Stage 2/3), and major vascular complications at 30 days. The primary effectiveness endpoint was a composite of all-cause mortality, disabling stroke, and moderate or greater paravalvular leak measured at 6 months. TF, transfemoral; TAo, transaortic. 


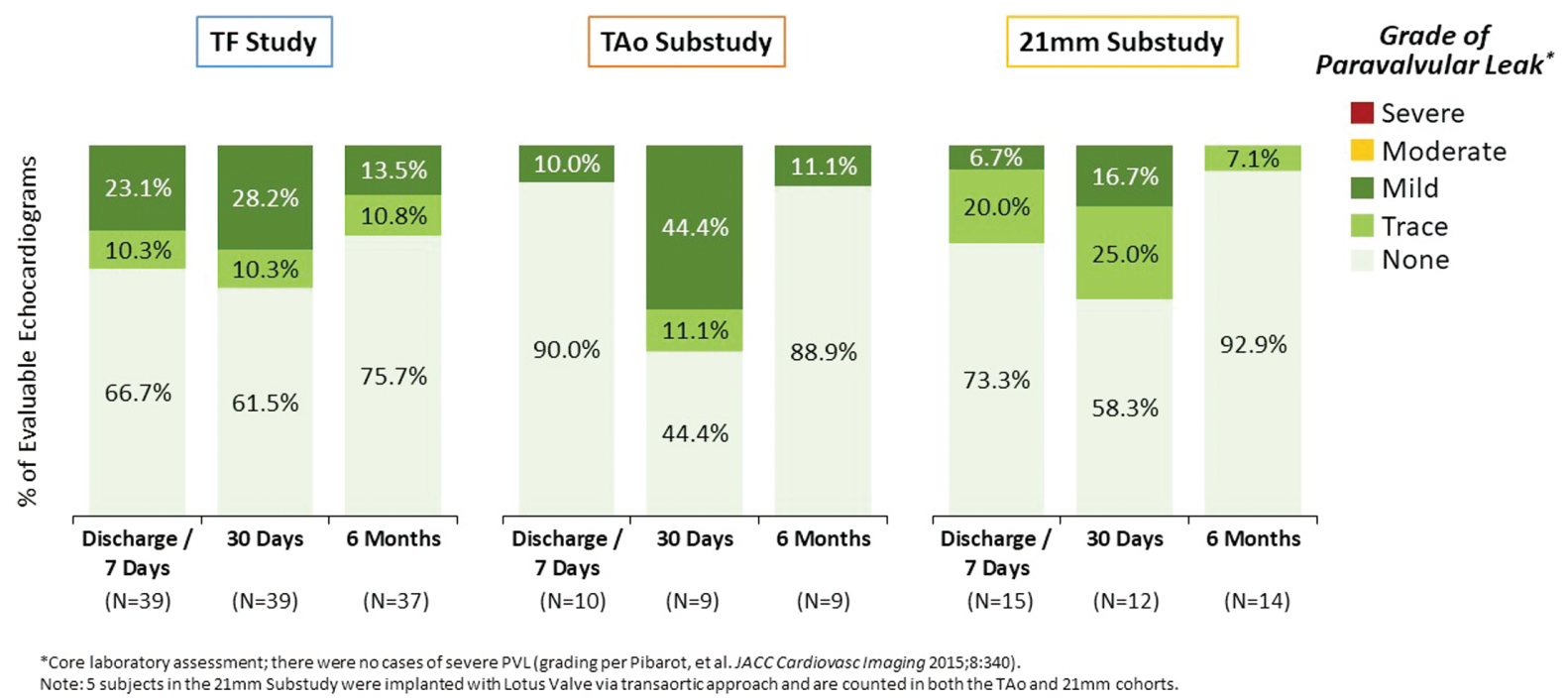

Figure 4. Degree of paravalvular leak (PVL) evaluated at discharge or 7 days and at the 30-day and 6-month follow-up visits. Echocardiographic measures were based on independent core laboratory assessment. TF, transfemoral; TAo, transaortic.

distributions were similar in the TAo and 21-mm substudies (Table 1). The most commonly implanted valve size was $23 \mathrm{~mm}$; only 4 subjects were treated with a $27-\mathrm{mm}$ valve.

\section{Clinical and Echocardiographic Outcomes}

Successful vascular access, device delivery, and deployment of the LOTUS valve, and successful retrieval of the delivery system, were achieved in all REPRISE Japan subjects (Table 1). Repositioning of the valve was attempted in 17 subjects in the TF study (42.5\%), and was successful (i.e., partial or complete resheathing of the LOTUS valve in the catheter and redeployment in a more accurate position within the aortic valve annulus) for the last valve attempted in $100.0 \%$ of subjects. In the TAo substudy, repositioning was attempted in 5 of 10 subjects $(50.0 \%)$ and was successful in all of them. In the 21-mm substudy, repositioning was attempted in 5 of 15 subjects $(33.3 \%)$ and was successful in $100 \%$. Retrieval of the valve (i.e., complete resheathing of the LOTUS valve in the catheter and removal from the body) was attempted in 3 subjects in the TF study and 1 subject in the $21-\mathrm{mm}$ substudy; the valve was successfully retrieved in all cases.

Thirty-day clinical follow-up was available for all REPRISE Japan subjects. The 30 -day primary safety endpoint rate was $15 \%$ among subjects in the TF study (Table 2; Figure 3). The rate of the primary safety endpoint was higher in the TAo and 21-mm substudies $(60 \%$ and $26.7 \%$, respectively), due primarily to higher rates of bleeding events in these cohorts.

At 6 months after the procedure, clinical follow-up was available for $95 \%$ of subjects in the TF study, $100 \%$ of subjects in the TAo substudy, and $93.3 \%$ of subjects in the 21-mm substudy (Figure 2). The primary effectiveness endpoint occurred in $5.3 \%$ of subjects the TF study, $10 \%$ of subjects in the TAo substudy, and $7.1 \%$ of subjects in the 21-mm substudy (Figure 3). The degree of moderate or greater PVL at 6 months was also analyzed separately as a secondary endpoint. Across the TF, TAo, and 21-mm cohorts, no subjects exhibited more than mild PVL at 6 months (Figure 4).

There were no deaths within 30 days. At 6 months, 1 cardiovascular death in the TF study and 1 cardiovascular death in the TAo substudy were observed. The rate of disabling stroke through 6 months was $2.5 \%(1 / 40)$ in the TF study, $0.0 \%(0 / 10)$ in the TAo cohort, and $6.7 \%(1 / 15)$ in the 21-mm cohort. None of the subjects in REPRISE Japan experienced a myocardial infarction through 6 months. The rate of hospitalization for valve-related symptoms or worsening congestive heart failure was $0.0 \%$ in the TF study and $21-\mathrm{mm}$ cohort, and $10 \%$ in the TAo cohort (1/10 subjects). No subjects required reintervention for valve-related dysfunction. There was no incidence of periprocedural annular rupture in REPRISE Japan, and no cases of valve malpositioning, endocarditis, or valve thrombosis through 30 days (there was 1 case of endocarditis and 1 case of thrombosis in the TAo cohort at 6 months). The 30 -day PPI rate was $22.5 \%$ in the TF cohort, $20 \%$ in the TAo cohort, and $13.3 \%$ in the $21-\mathrm{mm}$ cohort. Between 30 days and 6 months, pacemaker implantation was required in 1 additional subject from the TAo cohort (Table 1).

Transthoracic echocardiography assessment demonstrated improvement in valve hemodynamics after TAVR for all REPRISE Japan subjects, which was sustained at 6 months (Figure 5). In the TF study, aortic valve area (effective orifice area) improved from a mean $( \pm S D)$ of $0.6 \pm 0.2 \mathrm{~cm}^{2}$ at baseline to $1.4 \pm 0.3 \mathrm{~cm}^{2}$ at discharge or 7 days and $1.5 \pm 0.4 \mathrm{~cm}^{2}$ at 6 months. Mean $( \pm \mathrm{SD})$ aortic valve gradient in the TF cohort declined from $57.3 \pm 20.2 \mathrm{mmHg}$ at baseline to $13.2 \pm 4.8 \mathrm{mmHg}$ at discharge or 7 days, and remained at $13.4 \pm 5.7 \mathrm{mmHg}$ at 6 months. The hemodynamic improvements observed in the TAo and 21-mm substudies were of a similar magnitude (Figure 5).

\section{NYHA Functional Status}

At baseline, most REPRISE Japan subjects were catego- 


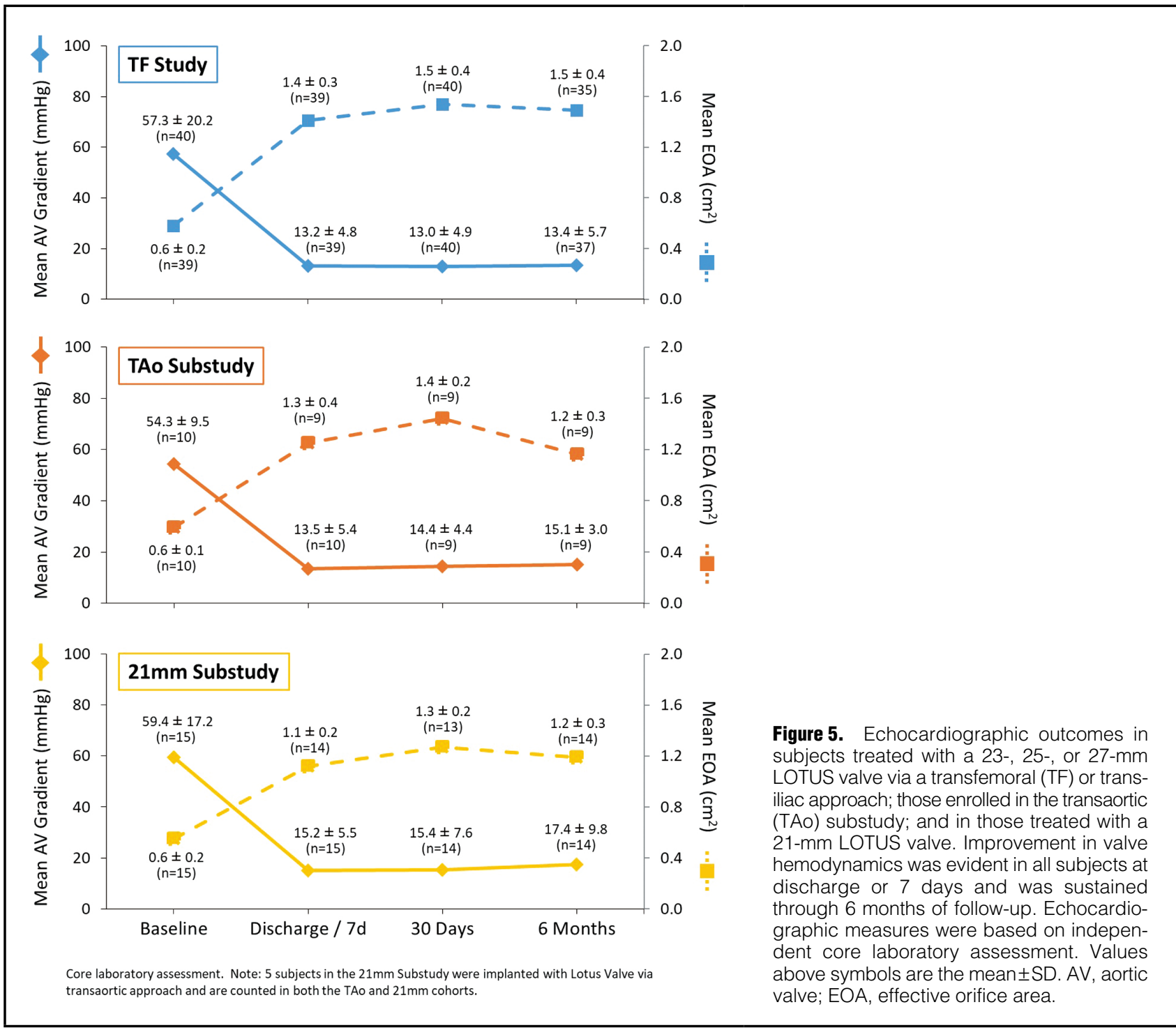

rized with an NYHA functional class of III or higher (Figure 6). Fifty percent of subjects in the TF study were NYHA Class III at baseline; an additional $2.5 \%$ were Class IV. In the TAo and $21-\mathrm{mm}$ substudies, $40.0 \%$ and $46.7 \%$ of subjects were Class III at baseline. Overall, substantial improvement in NYHA functional class was observed at discharge or 7 days and was maintained through 6 months of follow-up. Among surviving subjects, improvement of at least one class relative to baseline was observed in $97.3 \%$ of the TF cohort, $100 \%$ of the TAo cohort, and $85.7 \%$ of the 21-mm cohort.

\section{Discussion}

\section{Key Findings From REPRISE Japan}

Clinical outcomes from the REPRISE Japan study confirm the safety and effectiveness of TAVR with the LOTUS valve in Japanese subjects with severe symptomatic calcific aortic stenosis at extreme or high surgical risk. In the main TF study, the primary safety endpoint, a composite of allcause mortality, stroke, life-threatening or major bleeding events, acute kidney injury (Stage 2/3), and major vascular complications at 30 days, occurred in $15 \%$ of subjects. There were no deaths in the TF study within 30 days, the rate of disabling stroke was low $(2.5 \%)$, and no TF subjects required reintervention or hospitalization for valve-related symptoms or worsening heart failure.

The study also included important subanalyses of subjects treated via a TAo approach and, for the first time, subjects treated with the smaller 21-mm LOTUS valve. Both these cohorts had good functional outcomes, with only 1 cardiovascular death (TAo cohort) and 1 disabling stroke (21-mm cohort) through 6 months of follow-up. However, the rate of the primary safety endpoint was higher in the both the TAo and 21-mm substudies $(60 \%$ and $26.7 \%$, respectively), due primarily to higher rates of bleeding events in these cohorts. This is not unexpected, because other studies have also shown higher rates of bleeding complications with the TAo compared with TF approach. ${ }^{15,16}$ Bleeding complications can usually be managed in a controlled manner to optimize procedural success. The substudy analyses are somewhat limited by the very small number of subjects in these cohorts; further investigation will provide more information on clinical 


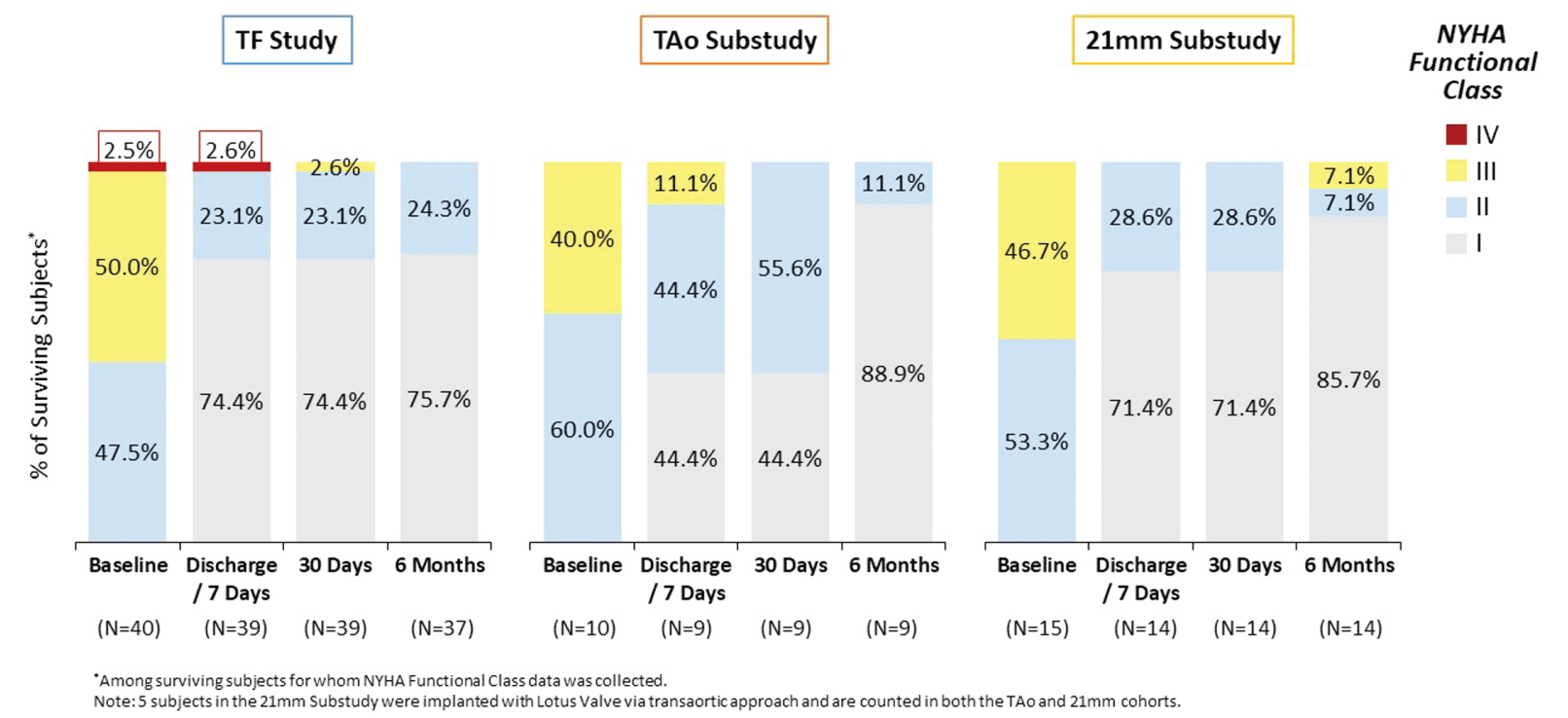

Figure 6. New York Heart Association (NYHA) Functional Class in subjects treated with a 23-, 25-, or 27-mm LOTUS valve via a transfemoral (TF) or transiliac approach; those enrolled in the transaortic (TAo) substudy; and in those treated with a 21-mm LOTUS valve. At baseline, most subjects were categorized with an NYHA functional class of III or higher. Overall, substantial improvement in NYHA functional class was observed at discharge or 7 days and was maintained through 6 months of follow-up.

outcomes in these groups.

Based on core laboratory assessment of echocardiographic data, hemodynamic measures improved for all subjects in REPRISE Japan. In the TF study and both the TAo and 21-mm cohorts, subjects exhibited increased mean effective orifice areas and reduced mean aortic valve gradients at discharge or 7 days and through 6 months of follow-up. As expected with a smaller prosthesis, subjects treated with the $21-\mathrm{mm}$ valve had slightly higher gradients.

\section{REPRISE Japan in Context With Contemporary Studies}

Although the REPRISE Japan study is not a randomized study, and thus lacks a direct comparator for the LOTUS valve, its findings may be evaluated in the context of contemporary TAVR studies. The safety outcomes from REPRISE Japan are similar to those observed in other Japanese TAVR subjects. For example, in the Transfemoral \& Transapical Placement of Aortic Balloon Expandable Transcatheter Valves Trial (Japan) (PREVAIL-J) study, a single-arm prospective non-randomized clinical trial evaluating the Edwards SAPIEN XT valve in 64 Japanese subjects, the combined safety endpoint at 30 days (all-cause mortality, major stroke, life-threatening or disabling bleeding, acute kidney injury Stage 3, periprocedural myocardial infarction, major vascular complication, and further intervention due to valve dysfunction) occurred in $25 \%$ of subjects, and $4.7 \%$ of subjects required conversion to surgical valve replacement. ${ }^{17}$ The large Optimized Transcatheter Valvular InterventionTranscatheter Aortic Valve Implantation (OCEAN-TAVI) registry evaluated 1,613 Japanese patients treated with SAPIEN-XT, SAPIEN 3, or CoreValve. ${ }^{6}$ In the OCEANTAVI registry, the 30 -day mortality rate was $1.7 \%$ and the combined early non-safety endpoint (a combination of all-cause mortality, all stroke, life-threatening bleeding, acute kidney injury Stage 2-3, coronary obstruction requiring intervention, major vascular complication, and valve-related dysfunction requiring repeated TAVR or surgical aortic valve replacement) occurred in $15.1 \%$ of subjects. ${ }^{6}$ The Asian TAVR Registry, ${ }^{5}$ which evaluated the safety, efficacy, and clinical outcomes of 848 Asian patients who underwent TAVR with SAPIEN and CoreValve prostheses, had a 30 -day mortality rate of $2.5 \% ; 1.8 \%$ of subjects required conversion to open heart surgery and 14.6\% of subjects had experienced early safety events (i.e., cardiac death, stroke, bleeding events, vascular complications, acute kidney injury, and device success) at 30 days.

Comparatively, the rate of all-cause mortality at 30 days was higher in recent European and US TAVR studies, including the Placement of AoRTic TraNscathetER Valve Trial Edwards SAPIEN Transcatheter Heart Valve (PARTNER) 2B study (SAPIEN: 5.1\%; SAPIEN XT: $3.5 \%),{ }^{18}$ the CoreValve US High-risk study $(3.3 \%),{ }^{19}$ the Evolut R US study $(2.5 \%),{ }^{20}$ and REPRISE II $(4.2 \%)^{10}$ and III $(2.5 \%) .{ }^{12}$ In these Western study populations, the 30-day stroke rate ranged from $3.0 \%$ to $5.9 \%$; although the incidence of stroke at 30 days was somewhat higher in the present REPRISE Japan TF study (3/40; 7.5\%), this may simply be a chance observation, amplified by the smaller patient population.

An analysis comparing TAVR in Asian patients enrolled in PREVAIL-J to a cohort of patients from France observed a numerically higher incidence of procedural complications among Asian patients. ${ }^{7}$ Some of the concerns related to vascular access, valve positioning, and valve sizing may be mitigated by certain design features of the LOTUS valve, namely its mechanical expansion, repositionability, retrievability, and integrated sealing skirt, which are discussed in more detail below. The rate of major vascular complications in the present REPRISE Japan TF study $(2.5 \%)$ compares favorably with other studies of the LOTUS valve (REPRISE II: $2.5 \%$; REPRISE III: $7.0 \%),{ }^{10,12}$ and was lower than the rates observed with 
SAPIEN and CoreValve prostheses in both Japanese studies (PREVAIL-J: 9.4\%; Asian TAVR registry: 5.0\%), and in Western populations (PARTNER 2B: 15.5\%-22.2\%; CoreValve US High-risk study: 5.9\%). ${ }^{18,19}$

\section{Advantages and Disadvantages of TAVR With the LOTUS Valve}

In any TAVR procedure, correct positioning and deployment of the valve are of utmost importance to prevent adverse outcomes such as embolization and to limit the occurrence of PVL., ${ }^{921}$ A major advantage of the LOTUS valve is its controlled delivery. The design of the LOTUS valve promotes smooth deployment through gradual and continuous mechanical expansion, lowering the risk for aortic root rupture that may accompany balloon-expandable valves. ${ }^{22}$ In the present study there were no annular ruptures with the LOTUS valve, compared with a rupture rate of $1.6 \%$ in PREVAIL- $\mathrm{J}^{17}$ and $1.7 \%$ in the Kyoto University-related hospital Transcatheter Aortic Valve Implantation (K-TAVI) registry, ${ }^{8}$ both of which studied the balloon-expandable SAPIEN XT valve. The LOTUS valve is repositionable and fully retrievable, which allows the operator to fully assess valve positioning prior to release. This is critical for ensuring correct sizing and placement, and helps minimize the risk of malpositioning, coronary occlusion, valve migration, and embolization. In the present REPRISE Japan study, there were no cases of valve malpositioning (valve migration, valve embolization, or ectopic valve deployment).

Perhaps more importantly, the repositionability and retrievability of the LOTUS valve enables operators to make any necessary corrections in positioning to minimize PVL. The LOTUS valve also has an integrated sealing skirt designed to further reduce PVL by filling the space between the native annulus and the prosthetic valve frame, which is particularly helpful in patients with bicuspid valves and/or heavily calcified annuli. PVL rates in the present REPRISE Japan study were better than in contemporary studies with self-expanding or balloon-expandable valves. At no time during the study did any subjects exhibit greater than mild PVL. Comparatively, the percentage of subjects with moderate or greater PVL ranged from $18.8 \%$ in PREVAIL$\mathrm{J}^{17}$ to $1.2 \%$ in TF subjects from the K-TAVI registry. ${ }^{8}$

Because the valve functions early in deployment, rapid pacing is not required, making it suitable for patients in whom hemodynamic compromise is a concern. However, because the LOTUS valve sheath is slightly larger than other transcatheter valve systems on the market, patients with very small vasculature may experience difficulties. A more flexible and lower-profile catheter has been developed, which, when used in conjunction with the trifold expandable iSleeve introducer (pending approval in Japan), may minimize vessel trauma and facilitate vascular access in such patients.

The LOTUS valve is known to have a higher PPI rate than contemporary second-generation TAVR valves. ${ }^{12,23}$ Although the rate of new PPI in the present REPRISE Japan study ( $22.5 \%$ in the TF study overall) is comparable to that observed with self-expanding valves in the Asian TAVR and OCEAN-TAVI registries $(19.4 \%$ and $23.5 \%$, respectively), it is higher than the rates observed with balloon-expandable valves $\left(4.0 \%\right.$ and $7.1 \%$, respectively). ${ }^{\mathbf{5}, 6}$ Post hoc analyses have shown that valve oversizing (e.g., $>10 \%$ annular overstretch) and a greater implantation depth are associated with a higher rate of PPI in patients treated with the LOTUS valve. ${ }^{24,25}$ Similarly, a post hoc analysis of data from the REepositionable Lotus Valve System-POst-Market EvaluatioN of Real WorlD Clinical Outcomes (RESPOND) post-market registry demonstrated that PPI was highly correlated with valve implantation depth. ${ }^{26}$ In the RESPOND study, the overall PPI rate at 30 days was 30\% among participants treated with the classic LOTUS valve. ${ }^{27}$ In the RESPOND Extension cohort, 50 additional subjects were treated with the LOTUS valve with Depth Guard, which prevented deep implantation of the valve and yielded a lower PPI rate (18\%) at 30 days. ${ }^{28}$

The current iteration of the LOTUS Valve, LOTUS Edge, was approved for use in Japan as of December 2019. LOTUS Edge retains the key features of controlled mechanical expansion, repositionability, retrievability, and an adaptive seal to minimize PVL. Design modifications include an improved catheter with increased flexibility, a reduced profile, and an optimized preshaped curve. The LOTUS Edge valve also features 1-view locking with radiopaque markers and Depth Guard technology, designed to limit the depth of implant, thereby reducing interactions with the left ventricular outflow tract to minimize the risk of conduction disorders. In an interim analysis of the currently enrolling RESPOND EDGE post-market study (NCT04009720), subjects treated with LOTUS Edge had a 30 -day PPI rate of $18 \%$ at discharge. ${ }^{29}$ As implanters continue to gain experience with the combination of modifications to implant technique and valve design, the PPI rate with LOTUS may decline.

\section{Conclusions}

Data from the REPRISE Japan study confirm the safety and effectiveness of TAVR with the LOTUS valve in Japanese clinical practice. Subjects exhibited excellent valve hemodynamics and minimal PVL. Features of the LOTUS valve, including deployment via gradual mechanical expansion by either a TF or TAo route and its repositionability and retrievability, may make it particularly well-suited to addressing challenges related to access, positioning, and sizing in a Japanese patient population. Attention to patient selection and modifications to implant technique and valve design may help minimize complications, including the need for PPI.

\section{Acknowledgments}

The authors thank Koichi Maeda, MD, PhD (Department of Cardiovascular Surgery, Osaka University Graduate School of Medicine) for critical review of the manuscript, Hong Wang, MS (Boston Scientific Corporation) for statistical analysis, and MaryEllen Carlile Klusacek, $\mathrm{PhD}$ (Boston Scientific Corporation) for help with manuscript preparation.

\section{Sources of Funding}

The REPRISE Japan study was sponsored and funded by Boston Scientific Corporation.

\section{Disclosures}

T.G. is a proctor for Edwards Lifesciences. L.I. is an employee of and shareholder in Boston Scientific. Y.S. has received research funding from Edwards Lifesciences, Medtronic Japan, and Livanova Corporation; scholarship (incentive) grants from Edwards Lifesciences, Otsuka Pharmaceutical Co., Konishi Medical Instruments Co., Abiomed Japan, and Abbott Medical Japan; patent royalties/licensing fees from Terumo Corporation; and is a shareholder in Qualips Co. Ltd.

Y.S. and K.H. are members of the Editorial Board of Circulation 
Journal. The remaining authors have no conflicts of interest to declare.

\section{IRB Information}

This study was approved by the ethics committee of Mirai Iryo Research Center Inc. (Reference no. 024-14-25).

\section{Data Availability}

Individual deidentified participant data will not be shared for this study. Boston Scientific's Data Sharing Policy can be accessed at http://www.bostonscientific.com/en-US/data-sharing-requests.html.

\section{References}

1. Makkar RR, Fontana GP, Jilaihawi H, Kapadia S, Pichard AD, Douglas PS, et al. Transcatheter aortic-valve replacement for inoperable severe aortic stenosis. $N$ Engl J Med 2012; 366: $1696-1704$.

2. Thourani VH, Kodali S, Makkar RR, Herrmann HC, Williams $\mathrm{M}$, Babaliaros V, et al. Transcatheter aortic valve replacement versus surgical valve replacement in intermediate-risk patients: A propensity score analysis. Lancet 2016; 387: 2218-2225.

3. Leon MB, Smith CR, Mack MJ, Makkar RR, Svensson LG, Kodali SK, et al. Transcatheter or surgical aortic-valve replacement in intermediate-risk patients. $N$ Engl J Med 2016; 374: $1609-1620$.

4. Smith CR, Leon MB, Mack MJ, Miller DC, Moses JW, Svensson LG, et al. Transcatheter versus surgical aortic-valve replacement in high-risk patients. $N$ Engl J Med 2011; 364: 2187-2198.

5. Yoon SH, Ahn JM, Hayashida K, Watanabe Y, Shirai S, Kao $\mathrm{HL}$, et al. Clinical outcomes following transcatheter aortic valve replacement in Asian population. JACC Cardiovasc Interv 2016; 9: $926-933$.

6. Yamamoto M, Watanabe Y, Tada N, Naganuma T, Araki M, Yamanaka F, et al. Transcatheter aortic valve replacement outcomes in Japan: Optimized CathEter vAlvular iNtervention (OCEAN) Japanese multicenter registry. Cardiovasc Revasc Med 2019; 20: $843-851$.

7. Watanabe Y, Hayashida K, Takayama M, Mitsudo K, Nanto S, Takanashi S, et al. First direct comparison of clinical outcomes between European and Asian cohorts in transcatheter aortic valve implantation: The Massy study group vs. the PREVAIL JAPAN trial. J Cardiol 2015; 65: 112-116.

8. Takimoto S, Saito N, Minakata K, Shirai S, Isotani A, Arai Y, et al. Favorable clinical outcomes of transcatheter aortic valve implantation in Japanese patients: First report from the postapproval K-TAVI registry. Circ J 2017; 81: 103-109.

9. Kanzaki H. Several concerns in transcatheter aortic valve replacement in Japanese elderly. J Cardiol 2015; 65: 89-90.

10. Meredith IT, Walters D, Dumonteil N, Worthley SG, Tchétché D, Manoharan G, et al. Transcatheter aortic valve replacement for severe symptomatic aortic stenosis using a repositionable valve system: 30-day primary endpoint results from the REPRISE II study. Am J Coll Cardiol 2014; 64: 1339-1348.

11. Meredith IT, Worthley SG, Whitbourn RJ, Antonis P, Montarello JK, Newcomb AE, et al. Transfemoral aortic valve replacement with the repositionable LOTUS valve system in high surgical risk patients: The REPRISE I study. EuroIntervention 2014; 9: $1264-1270$.

12. Feldman TE, Reardon MJ, Rajagopal V, Makkar RR, Bajwa TK, Kleiman NS, et al. Effect of mechanically expanded vs selfexpanding transcatheter aortic valve replacement on mortality and major adverse clinical events in high-risk patients with aortic stenosis: The REPRISE III randomized clinical trial. JAMA 2018; 319: 27-37.

13. Gooley R, Lockwood S, Antonis P, Meredith IT. The SADRA LOTUS valve system: A fully repositionable, retrievable prosthesis. Minerva Cardioangiol 2013; 61: 45-52.

14. Kappetein AP, Head SJ, Genereux P, Piazza N, van Mieghem NM, Blackstone EH, et al. Updated standardized endpoint definitions for transcatheter aortic valve implantation: The Valve Academic Research Consortium-2 consensus document. Am J Coll Cardiol 2012; 60: 1438-1454.

15. Chollet T, Marcheix B, Boudou N, Elbaz M, Campelo-Parada
F, Bataille V, et al. Propensity-matched comparison of clinical outcomes after transaortic versus transfemoral aortic valve replacement. EuroIntervention 2018; 14: 750-757.

16. Arai T, Romano M, Lefèvre T, Hovasse T, Farge A, Le Houerou $\mathrm{D}$, et al. Direct comparison of feasibility and safety of transfemoral versus transaortic versus transapical transcatheter aortic valve replacement. JACC Cardiovasc Interv 2016; 9: 2320-2325.

17. Sawa Y, Takayama M, Mitsudo K, Nanto S, Takanashi S, Komiya T, et al. Clinical efficacy of transcatheter aortic valve replacement for severe aortic stenosis in high-risk patients: The PREVAIL Japan trial. Surg Today 2015; 45: 34-43.

18. Webb JG, Doshi D, Mack MJ, Makkar R, Smith CR, Pichard $\mathrm{AD}$, et al. A randomized evaluation of the SAPIEN XT transcatheter heart valve system in patients with aortic stenosis who are not candidates for surgery. JACC Cardiovasc Interv 2015; 8: $1797-1806$.

19. Adams DH, Popma JJ, Reardon MJ, Yakubov SJ, Coselli JS, Deeb GM, et al. Transcatheter aortic-valve replacement with a self-expanding prosthesis. $N$ Engl J Med 2014; 370: $1790-$ 1798.

20. Popma JJ, Reardon MJ, Khabbaz K, Harrison JK, Hughes GC, Kodali S, et al. Early clinical outcomes after transcatheter aortic valve replacement using a novel self-expanding bioprosthesis in patients with severe aortic stenosis who are suboptimal for surgery: Results of the Evolut R U.S. study. JACC Cardiovasc Interv 2017; 10: $268-275$.

21. Makkar RR, Jilaihawi H, Chakravarty T, Fontana GP, Kapadia $\mathrm{S}$, Babaliaros V, et al. Determinants and outcomes of acute transcatheter valve-in-valve therapy or embolization: A study of multiple valve implants in the U.S. PARTNER trial (Placement of AoRTic TraNscathetER Valve Trial Edwards SAPIEN Transcatheter Heart Valve). Am J Coll Cardiol 2013; 62: 418-430.

22. Okuno T, Asami M, Heg D, Lanz J, Praz F, Hagemeyer D, et al. Impact of left ventricular outflow tract calcification on procedural outcomes after transcatheter aortic valve replacement. JACC Cardiovasc Interv 2020; 13: 1789-1799.

23. Seeger J, Gonska B, Rottbauer W, Wöhrle J. Outcome with the repositionable and retrievable boston scientific LOTUS valve compared with the balloon-expandable Edwards Sapien 3 valve in patients undergoing transfemoral aortic valve replacement. Circ Cardiovasc Interv 2017; 10: e004670.

24. Dumonteil N, Meredith IT, Blackman DJ, Tchétché D, HildickSmith D, Spence MS, et al. Insights into the need for permanent pacemaker following implantation of the repositionable LOTUS ${ }^{\mathrm{TM}}$ valve for the transcatheter aortic valve replacement in 250 patients: Results from the REPRISE II trial with extended cohort. EuroIntervention 2017; 13: 796-803.

25. De Backer O, Götberg M, Ihlberg L, Packer E, Savontaus M, Nielsen NE, et al. Efficacy and safety of the LOTUS valve system for treatment of patients with severe aortic valve stenosis and intermediate surgical risk: Results from the Nordic LOTUSTAVR registry. Int J Cardiol 2016; 219: 92-97.

26. van Gils L, Wöhrle J, Hildick-Smith D, Bleiziffer S, Blackman DJ, Abdel-Wahab M, et al. Importance of contrast aortography with LOTUS transcatheter aortic valve replacement: A post hoc analysis from the RESPOND post-market study. JACC Cardiovasc Interv 2018; 11: 119-128.

27. Falk V, Wöhrle J, Hildick-Smith D, Bleiziffer S, Blackman DJ, Abdel-Wahab M, et al. Safety and efficacy of a repositionable and fully retrievable aortic valve used in routine clinical practice: The RESPOND Study. Eur Heart J 2017; 38: 3359-3366.

28. Mieghem NMV, Wöhrle J, Hildick-Smith D, Bleiziffer S, Blackman DJ, Abdel-Wahab M, et al. Use of a repositionable and fully retrievable aortic valve in routine clinical practice: The RESPOND Study and RESPOND Extension Cohort. JACC Cardiovasc Interv 2019; 12: 38-49.

29. Kharbanda R. Early clinical outcomes with a repositionable and fully retrievable aortic valve in the first 50 patients enrolled in the RESPOND EDGE registry presented at: Transcatheter Valve Therapeutics Structural Heart Summit Online, June 2020.

\section{Supplementary Files}

Please find supplementary file(s);

http://dx.doi.org/10.1253/circj.CJ-20-0064 